\title{
Existence of global weak solutions for a viscous 2D bilayer Shallow Water model
}

\section{Existence de solutions faibles globales d'un modèle bicouche bidimensionnel de Saint-Venant}

\author{
Gladys Narbona-Reina ${ }^{a}$ Jean De Dieu Zabsonré ${ }^{b}$ \\ ${ }^{a}$ Universidad de Sevilla, Dpto. Matemática Aplicada I, Avda. Reina Mercedes 2, 41012 Sevilla (Spain) \\ ${ }^{\mathrm{b}}$ Université Polytechnique de Bobo-Dioulasso, Institut des Sciences Exactes et Appliquées, 01 BP 1091 Bobo 01
}

\begin{abstract}
We consider a system composed by two immiscible fluids in two-dimensional space that can be modelized by a bilayer Shallow Water equations with extra friction terms and capillary effects. We give an existence theorem of global weak solutions in a periodic domain.
\end{abstract}

\section{Résumé}

Nous considérons un système composé par deux fluides immiscibles dans un domaine bi-dimensionnel pouvant être représenté par un modèle bicouche visqueux de Saint-Venant avec des termes de friction additionnels et des effets de capillarité. Nous donnons un théorème d'existence de solutions faibles globales dans un domaine périodique.

\section{Version française abrégée}

Dans cette note, nous nous intéressons à l'étude de l'existence de solutions faibles globales en temps d'un modèle bicouche visqueux de Saint-Venant dérivé dans [6]. Notons que dans le cas d'une couche, dans [1] et [4] les auteurs ont obtenu l'existence de solutions faibles grâce à une nouvelle entropie (BD) introduite premièrement par Bresch et Desjardins dans [1]. On peut trouver d'autres résultats sur l'existence de solutions pour des modèles bicouche de Saint-Venant dans [3] et [5]. Dans ces modèles, les termes couplant les deux fluides compliquent le passage à la limite. Dans [7] une étude du modèle bicouche mais où les termes de friction ont été simplifiés a été faite. Les termes de friction couplant les deux couches dans le

Email addresses: gnarbona@us.es (Gladys Narbona-Reina), jzabsonre@gmail.com (Jean De Dieu Zabsonré). 
présent cas sont plus difficiles à contrôler et compliquent également le passage à la limite dans les suites de solutions approchées. Notons que les termes de viscosité ici sont de la forme $\operatorname{div}\left(\mathrm{h}_{\mathrm{i}} \mathrm{D}\left(\mathrm{u}_{\mathrm{i}}\right)\right)$ et permettent comme dans [1], [4] et [6] d'avoir une plus grande régularité sur les hauteurs.

Plus précisement, nous considérons dans un domaine périodique bi-dimensionnel le modèle (1)-(4) cidessous. Pour ce modèle, nous obtenons le théorème suivant :

Théorème 0.1 Il existe une solution faible globale de (1)-(4) satisfaisant les inégalités d'énergies.

\section{Introduction}

In this note, we are interested in the study of the existence of global weak solution for a 2D bilayer Shallow-Water model derived in [6]. In the one layer case with degenerate viscous term the global existence of weak solutions is obtained in [1] and [4] thanks to a new entropy inequality namely BD entropy derived first by Bresch and Desjardins in [1]. Notice that other bilayer shallow water models is studied in [3] and [5]. The main difficulty in these papers arises from the terms coupling the two layers. We can also find in [7] the theoretical study of the bilayer model but where the friction terms have been simplified. The friction terms considered here are more difficult to control and to pass to the limit in the weak formulation. Such work will be the aim of this paper.

After these lines we introduce the model to study and we state how a weak solutions is defined for our system. In the Section 2 we show the energies inequalities that allow us to prove the stability of weak solution, for their construction one can follow the lines of [2]. The existence result is presented in the last section, where we also give the schedule of the proof.

We consider in a two-dimensional periodic domain $\Omega$, a system composed by two layers of immiscible fluids with different and constant densities $\rho_{i}$ and viscosities $\mu_{i}$, for $i=1,2$. The unknowns are the velocities $v_{i}$ and the thickness $h_{i}$, respectively. The index 1 design the deeper layer and the index 2 , the upper layer of the flow. We assume that the friction coefficient at the bottom $\alpha$ and the tension coefficients $\alpha_{1}, \alpha_{2}$ (on the interface and on the free surface, respectively) are positive. The model reads as follows:

$$
\begin{aligned}
& \partial_{t} h_{1}+\operatorname{div}\left(h_{1} v_{1}\right)=0 \\
& \rho_{1} \partial_{t}\left(h_{1} v_{1}\right)+\rho_{1} \operatorname{div}\left(h_{1} v_{1} \otimes v_{1}\right)-2 \mu_{1} \operatorname{div}\left(h_{1} D\left(v_{1}\right)\right) \\
& \quad+\rho_{1} g h_{1} \nabla h_{1}+\rho_{2} g h_{1} \nabla h_{2}+\delta^{-1} \gamma\left(\beta \frac{\alpha h_{1}}{6 \mu_{1}} v_{1}+\left(v_{1}-v_{2}\right)\right) \\
& \quad+\beta \alpha\left(v_{1}+\delta^{-1} \frac{\gamma h_{1}}{6 \mu_{1}}\left(v_{1}-v_{2}\right)\right)-\alpha_{1} h_{1} \nabla\left(\Delta h_{1}\right)-\alpha_{2} h_{1} \nabla\left(\Delta h_{2}\right)=0 \\
& \quad \\
& \partial_{t} h_{2}+\operatorname{div}\left(h_{2} v_{2}\right)=0 ; \\
& \rho_{2} \partial_{t}\left(h_{2} v_{2}\right)+\rho_{2} \operatorname{div}\left(h_{2} v_{2} \otimes v_{2}\right)-2 \mu_{2} \operatorname{div}\left(h_{2} D\left(v_{2}\right)\right) \\
& \quad+\rho_{2} g h_{2} \nabla h_{2}+\rho_{2} g h_{2} \nabla h_{1}-\delta^{-1} \gamma\left(\beta \frac{\alpha h_{1}}{6 \mu_{1}} v_{1}+\left(v_{1}-v_{2}\right)\right)-\alpha_{2} h_{2} \nabla\left(\Delta\left(h_{1}+h_{2}\right)\right)=0
\end{aligned}
$$

with initial conditions: $h_{i \mid t=0}=h_{i_{0}} \geq 0, \quad h_{i} v_{i \mid t=0}=m_{i_{0}}$, for which we assume the following regularity: $h_{i_{0}} \in L^{2}(\Omega), \quad \nabla h_{i_{0}} \in\left(L^{2}(\Omega)\right)^{2}, \quad \nabla \sqrt{h_{i_{0}}} \in\left(L^{2}(\Omega)\right)^{2}, \quad \frac{\left|m_{i_{0}}\right|^{2}}{h_{i_{0}}} \in L^{1}(\Omega), \quad \log { }_{-}\left(h_{i_{0}}\right) \in L^{1}(\Omega)$. The functions $\beta=\beta\left(h_{1}\right), \delta=\delta\left(h_{1}, h_{2}\right)$ and $\gamma=\gamma\left(h_{1}, h_{2}\right)$ are the drag coefficients given by: $\beta\left(h_{1}\right)=\left(1+\frac{\alpha}{3 \mu_{1}} h_{1}\right)^{-1}, \delta=\frac{3 \mu_{1} \mu_{2}+\gamma \mu_{2} h_{1}+\gamma \mu_{1} h_{2}}{3 \mu_{1} \mu_{2}}$ and $\gamma=\frac{h_{1} h_{2}}{\frac{\rho_{1}}{\rho_{2}} h_{1}+\frac{\rho_{2}}{\rho_{1}} h_{2}}$. The terms $D(v)=\frac{\nabla v+\nabla^{t} v}{2}$ 
and $A(v)=\frac{\nabla v-\nabla^{t} v}{2}$ are respectively the deformation and the vorticity tensors.

Definition 1.1 We shall say that $\left(h_{1}, h_{2}, v_{1}, v_{2}\right)$ is a weak solution of (1)-(4) if (1) and (3) hold in $\left(\mathcal{D}^{\prime}(0, T) \times \Omega\right)^{2} ; h_{1_{\mid t=0}}=h_{1}^{0} \geq 0$ and $h_{2 \mid t=0}=h_{2}^{0} \geq 0$ in $\mathcal{D}^{\prime}(\Omega)$, the following assumptions are satisfied:

$$
\begin{array}{r}
h_{i} \in L^{\infty}\left(0, T ; L^{2}(\Omega)\right) ; \nabla h_{i}, \sqrt{h_{i}} v_{i} \in L^{\infty}\left(0, T ;\left(L^{2}(\Omega)\right)^{2}\right) ; v_{1} \in L^{2}\left(0, T ;\left(L^{2}(\Omega)\right)^{2}\right) ; \\
\sqrt{h_{i}} D\left(v_{i}\right) \in L^{2}\left(0, T ;\left(L^{2}(\Omega)\right)^{4}\right) ; \sqrt{B\left(h_{1}, h_{2}\right)}\left(v_{1}-v_{2}\right) \in L^{2}\left(0, T ;\left(L^{2}(\Omega)\right)^{2}\right) ; \\
\nabla \sqrt{h_{i_{n}}} \in L^{2}\left(0, T ; L^{2}(\Omega)^{2}\right) ; \Delta h_{i_{n}} \in L^{2}\left(0, T ; L^{2}(\Omega)\right) ;
\end{array}
$$

and for any $\varphi \in \mathcal{C}^{\infty}((0, T) \times \Omega)^{2}$ with $\varphi(T, \cdot)=0$, ( $\varphi$ with compact support), we have:

$$
\begin{aligned}
& -\int_{\Omega} \rho_{1} h_{1}^{0} v_{1}^{0} h_{1}^{0} \varphi(0, \cdot)-\int_{0}^{T} \int_{\Omega} \rho_{1} h_{1}^{2} v_{1} \partial_{t} \varphi+\rho_{1} \int_{0}^{T} \int_{\Omega} h_{1}^{2}\left(v_{1} \cdot \varphi\right) \operatorname{div} v_{1}-\rho_{1} \int_{0}^{T} \int_{\Omega}\left(h_{1} v_{1} \otimes h_{1} v_{1}\right): D(\varphi) \\
& \quad+2 \mu_{1} \int_{0}^{T} \int_{\Omega} h_{1}^{2}\left(D\left(v_{1}\right): D(\varphi)\right)+2 \mu_{1} \int_{0}^{T} \int_{\Omega} h_{1}\left(D\left(v_{1}\right):\left(\nabla h_{1} \otimes \varphi\right)\right)+\int_{0}^{T} \int_{\Omega} \delta^{-1} \gamma\left(\beta \frac{\alpha h_{1}}{6 \mu_{1}} v_{1}\right. \\
& \left.+\left(v_{1}-v_{2}\right)\right) h_{1} \varphi+\int_{0}^{T} \int_{\Omega} \beta \alpha\left(v_{1}+\delta^{-1} \gamma \frac{h_{1}}{6 \mu_{1}}\left(v_{1}-v_{2}\right)\right) h_{1} \varphi+\frac{1}{2} \rho_{1} g \int_{0}^{T} \int_{\Omega} h_{1}^{3} \operatorname{div} \varphi \\
& +\frac{1}{2} \rho_{1} g \int_{0}^{T} \int_{\Omega} h_{1}^{2}\left(\varphi \cdot \nabla h_{1}\right)+\rho_{2} g \int_{0}^{T} \int_{\Omega}^{T} h_{1}^{2}\left(\varphi \cdot \nabla h_{2}\right)+\alpha_{1} \int_{0}^{T} \int_{\Omega} h_{1}^{2} \Delta h_{1} \operatorname{div} \varphi \\
& \quad+2 \alpha_{1} \int_{0}^{T} \int_{\Omega} h_{1} \Delta h_{1}\left(\varphi \cdot \nabla h_{1}\right)+\alpha_{2} \int_{0} \int_{\Omega} h_{1}^{2} \Delta h_{2} \operatorname{div} \varphi+\alpha_{2} \int_{0} \int_{\Omega} h_{1}\left(\varphi \cdot \nabla h_{1}\right) \Delta h_{2}=0
\end{aligned}
$$

and

$$
\begin{aligned}
& -\int_{\Omega} \rho_{2} h_{2}^{0} v_{2}^{0} h_{2}^{0} \varphi(0, \cdot)-\int_{0}^{T} \int_{\Omega} \rho_{2} h_{2}^{2} v_{2} \partial_{t} \varphi+\rho_{2} \int_{0}^{T} \int_{\Omega} h_{2}^{2}\left(v_{2} \cdot \varphi\right) \operatorname{div} v_{2}-\rho_{2} \int_{0}^{T} \int_{\Omega}\left(h_{2} v_{2} \otimes h_{2} v_{2}\right): D(\varphi) \\
& \quad+2 \mu_{2} \int_{0}^{T} \int_{\Omega} h_{2}^{2}\left(D\left(v_{2}\right): D(\varphi)\right)+2 \mu_{2} \int_{0}^{T} \int_{\Omega} h_{2}\left(D\left(v_{2}\right):\left(\nabla h_{2} \otimes \varphi\right)\right)-\int_{0}^{T} \int_{\Omega} \delta^{-1} \gamma\left(\beta \frac{\alpha h_{1}}{6 \mu_{1}} v_{1}\right. \\
& \left.+\left(v_{1}-v_{2}\right)\right) h_{2} \varphi+\frac{1}{2} \rho_{2} g \int_{0}^{T} \int_{\Omega} h_{2}^{3} \operatorname{div} \varphi+\frac{1}{2} \rho_{2} g \iint_{0}^{T} \int_{\Omega} h_{2}^{2}\left(\varphi \cdot \nabla h_{2}\right)+\rho_{2} g \int_{0} \int_{\Omega} h_{2}^{2}\left(\varphi \cdot \nabla h_{1}\right) \\
& +\alpha_{2} \int_{0}^{T} \int_{\Omega} h_{2}^{2} \Delta\left(h_{1}+h_{2}\right) \operatorname{div} \varphi+2 \alpha_{2} \int_{0}^{T} \int_{\Omega} h_{2} \Delta\left(h_{1}+h_{2}\right)\left(\varphi \cdot \nabla h_{2}\right)=0 .
\end{aligned}
$$

\section{Energy estimates}

Proposition 2.1 Let $\left(h_{1}, h_{2}, v_{1}, v_{2}\right)$ be a solution of the system (1)-(4). Then, the following inequality holds: 


$$
\begin{aligned}
\frac{1}{2} \rho_{1} \frac{d}{d t} \int_{\Omega} h_{1}\left|v_{1}\right|^{2}+\frac{1}{2} \rho_{2} \frac{d}{d t} \int_{\Omega} h_{2}\left|v_{2}\right|^{2}+2 \mu_{1} \int_{\Omega} h_{1}\left(D\left(v_{1}\right): D\left(v_{1}\right)\right)+2 \mu_{2} \int_{\Omega} h_{2}\left(D\left(v_{2}\right): D\left(v_{2}\right)\right) \\
\quad+\frac{1}{2} g\left(\rho_{1}-\rho_{2}\right) \frac{d}{d t} \int_{\Omega}\left|h_{1}\right|^{2}+\int_{\Omega}\left(\delta^{-1} \gamma \beta \frac{\alpha h_{1}}{3 \mu_{1}}+\alpha \beta\right)\left|v_{1}\right|^{2}+\frac{1}{2} \rho_{2} g \frac{d}{d t} \int_{\Omega}\left|h_{1}+h_{2}\right|^{2} \\
\quad+\frac{1}{2}\left(\alpha_{1}-\alpha_{2}\right) \frac{d}{d t} \int_{\Omega}\left|\nabla h_{1}\right|^{2}+\frac{\alpha_{2}}{2} \frac{d}{d t} \int_{\Omega}\left|\nabla\left(h_{1}+h_{2}\right)\right|^{2}+\int_{\Omega} \delta^{-1} \gamma\left|v_{1}-v_{2}\right|^{2} \\
\leq \frac{1}{2} \frac{\rho_{1}}{\rho_{2}} \int_{\Omega} h_{1}\left|v_{1}\right|^{2}+\frac{1}{2} \frac{\rho_{2}}{\rho_{1}} \int_{\Omega} h_{2}\left|v_{2}\right|^{2} .
\end{aligned}
$$

Proposition 2.2 If we assume that $\left(h_{1}, h_{2}, v_{1}, v_{2}\right)$ is a smooth solution of system (1)-(4), then

$$
\begin{aligned}
& \frac{1}{2} \rho_{2} \frac{d}{d t} \int_{\Omega} h_{1}\left|\rho_{1} v_{1}+2 \mu_{1} \nabla \log h_{1}\right|^{2}+\frac{1}{2} \rho_{1} \frac{d}{d t} \int_{\Omega} h_{2}\left|\rho_{2} v_{2}+2 \mu_{2} \nabla \log h_{2}\right|^{2} \\
& \quad+\rho_{1} \rho_{2}\left(\frac{1}{2} g\left(\rho_{1}-\rho_{2}\right) \frac{d}{d t} \int_{\Omega}\left|h_{1}\right|^{2}+\frac{1}{2} \rho_{2} g \frac{d}{d t} \int_{\Omega}\left|h_{1}+h_{2}\right|^{2}+\left(\delta^{-1} \gamma \beta \frac{\alpha h_{1}}{3 \mu_{1}}+\alpha \beta\right)\left|v_{1}\right|^{2}\right) \\
& \quad+2 \mu_{2} \rho_{1} \rho_{2} \int_{\Omega} h_{2}\left(A\left(v_{2}\right): A\left(v_{2}\right)\right)+2 \mu_{1} \rho_{1} \rho_{2} \int_{\Omega} h_{1}\left(A\left(v_{1}\right): A\left(v_{1}\right)\right)+\rho_{1} \rho_{2} \int_{\Omega} \delta^{-1} \gamma\left|v_{1}-v_{2}\right|^{2} \\
& \quad+\frac{1}{2} \rho_{1} \rho_{2}\left(\alpha_{1}-\alpha_{2}\right) \frac{d}{d t} \int_{\Omega}\left|\nabla h_{1}\right|^{2}+\frac{1}{2} \alpha_{2} \rho_{1} \rho_{2} \frac{d}{d t} \int_{\Omega}\left|\nabla\left(h_{1}+h_{2}\right)\right|^{2}+2 \mu_{1} \rho_{1} \rho_{2} g \int_{\Omega}\left|\nabla h_{1}\right|^{2} \\
& +2 \mu_{2} \rho_{1} \rho_{2} g \int_{\Omega}\left|\nabla h_{2}\right|^{2}+2 \mu_{1} \alpha_{1} \rho_{2} \int_{\Omega}\left|\Delta h_{1}\right|^{2}+2 \mu_{2} \alpha_{2} \rho_{1} \int_{\Omega}\left|\Delta h_{2}\right|^{2}-2 \mu_{1} \alpha \rho_{2} \frac{d}{d t} \int_{\Omega} \log \left(\frac{h_{1}}{3 \mu_{1}+\alpha h_{1}}\right) \\
& +2 \mu_{1} \alpha \rho_{2} \int_{\Omega} \beta^{\prime}\left(h_{1}\right) v_{1} \nabla h_{1}+2 \rho_{2} g\left(\rho_{2} \mu_{1}+\rho_{1} \mu_{2}\right) \int_{\Omega} \nabla h_{1} \nabla h_{2}+2 \alpha_{2}\left(\rho_{2} \mu_{1}+\rho_{1} \mu_{2}\right) \int_{\Omega} \Delta h_{1} \Delta h_{2} \\
& +2 \mu_{1} \rho_{2} \int_{\Omega} \delta^{-1} \gamma\left(1+\beta \frac{\alpha h_{1}}{6 \mu_{1}}\right)\left(v_{1}-v_{2}\right) \cdot \nabla \log h_{1}+2 \mu_{1} \rho_{2} \int_{\Omega} \delta^{-1} \gamma \beta \frac{\alpha h_{1}}{6 \mu_{1}} v_{1} \cdot \nabla \log h_{1} \\
& -2 \mu_{2} \rho_{1} \int_{\Omega} \delta^{-1} \gamma \beta \frac{\alpha h_{1}}{6 \mu_{1}} v_{1} \cdot \nabla \log h_{2}-2 \mu_{2} \rho_{1} \int_{\Omega} \delta^{-1} \gamma\left(v_{1}-v_{2}\right) \cdot \nabla \log h_{2} \\
& \leq \frac{1}{6} \rho_{1}\left(\frac{3}{\rho_{2}}+\alpha\right) \int_{\Omega} h_{1}\left|v_{1}\right|^{2}+\frac{1}{2} \rho_{2}^{2} \int_{\Omega} h_{2}\left|v_{2}\right|^{2}+\frac{1}{3} \rho_{1} \alpha \int_{\Omega} h_{1}\left|\nabla h_{1}\right|^{2}
\end{aligned}
$$

Remark 1 The main difficulty in the proof of the two preceding ineqalities is located in the terms coming from the friction effects between the two fluids and between the lower fluid and the bottom. They are those we are going to focus on. The rest of the terms are controlled as in [7]. Note that:

- $F_{1}=2 \mu_{1} \rho_{2} \int_{\Omega} \delta^{-1} \gamma \beta \frac{\alpha h_{1}}{6 \mu_{1}} v_{1} \cdot \nabla \log h_{1}$. And we use the fact that $\delta^{-1} \leq 1, \beta \leq 1$ and $\gamma \leq \frac{\rho_{1}}{\rho_{2}} h_{1}$ to get:

$$
F_{1} \leq \frac{\rho_{1} \alpha}{6}\left(\int_{\Omega}^{\Omega} h_{1}\left|v_{1}\right|^{2}+\int_{\Omega} h_{1}\left|\nabla h_{1}\right|^{2}\right) .
$$

Next, since $\frac{\alpha \beta}{6 \mu_{1}} h_{1} \leq \frac{1}{2}$, we have

- $F_{2}=2 \mu_{1} \rho_{2} \int_{\Omega} \delta^{-1} \gamma\left(1+\beta \frac{\alpha h_{1}}{6 \mu_{1}}\right)\left(v_{1}-v_{2}\right) \cdot \nabla \log h_{1} \leq \frac{3}{2} \mu_{1} \rho_{2} \int_{\Omega} \delta^{-1} \gamma\left|v_{1}-v_{2}\right|^{2}+6 \mu_{1} \rho_{1} \int_{\Omega}\left|\nabla \sqrt{h_{1}}\right|^{2}$. 
And similarly the two last terms:

- $F_{3}=2 \mu_{2} \rho_{1} \int_{\Omega} \delta^{-1} \gamma \beta \frac{\alpha h_{1}}{6 \mu_{1}} v_{1} \cdot \nabla \log h_{2} \leq \mu_{2} \rho_{1} \int_{\Omega} \delta^{-1} \gamma \beta \frac{\alpha h_{1}}{6 \mu_{1}}\left|v_{1}\right|^{2}+2 \mu_{2} \rho_{2} \int_{\Omega}\left|\nabla \sqrt{h_{2}}\right|^{2}$
- $F_{4}=2 \mu_{2} \rho_{1} \int_{\Omega} \delta^{-1} \gamma\left(v_{1}-v_{2}\right) \cdot \nabla \log h_{2} \leq \frac{1}{2} \mu_{2} \rho_{1} \int_{\Omega} \delta^{-1} \gamma\left|v_{1}-v_{2}\right|^{2}+2 \mu_{2} \rho_{2}\left|\nabla \sqrt{h_{2}}\right|^{2}$.

\section{Main result}

Theorem 3.1 There exists a global weak solution $\left(h_{1}, h_{2}, v_{1}, v_{2}\right)$ of (1)-(4) satisfying the energy inequalities (8) and (9).

Proof. We consider a sequence of approximate weak solutions namely $\left(h_{1_{n}}, h_{2_{n}}, v_{1_{n}}, v_{2_{n}}\right)$ satisfying the two entropy inequalities. Such approximate solutions can be constructed with enough regularity along the lines of [2]. We assume that its initial data satisfy ( $C$ independent of $n): h_{i_{n}}^{0} \rightarrow h_{i}^{0}$ in $L^{1}(\Omega), h_{i_{n}}^{0} v_{i_{n}}^{0} \rightarrow$ $h_{i}^{0} v_{i}^{0}$ in $L^{1}(\Omega), \int_{\Omega} h_{i_{n}}^{0}\left|v_{i_{n}}^{0}\right|^{2}+\left|h_{i_{n}}^{0}\right|^{2}<C, \int_{\Omega} \nabla\left(h_{i_{n}}^{0}\right)^{1 / 2}<C i=0,1$. The proof of the Theorem 3.1 is the same as that built in [7]. We must only prove some additional strong convergences. We give first the preliminary convergence.

\section{Preliminary convergence.}

For $i=1,2$, we have $h_{i_{n}}$ bounded in $L^{2}\left(0, T ; H^{2}(\Omega)\right) \cap L^{\infty}\left(0, T ; H^{1}(\Omega)\right)$. Moreover $\partial_{t} h_{i_{n}}=-\operatorname{div}\left(h_{i_{n}} v_{i_{n}}\right)$ is bounded in $L^{2}\left(0, T ; H^{-1}(\Omega)\right)$. Thanks to the Sobolev's imbedding, we have $\forall s \in(0,1)$, $H^{1}(\Omega) \hookrightarrow H^{s}(\Omega), \quad H^{s}(\Omega) \subset \subset H^{-1}(\Omega), \quad H^{2}(\Omega) \hookrightarrow H^{1+s}(\Omega), \quad H^{1+s}(\Omega) \subset \subset H^{-1}(\Omega)$. Then, up to the extraction of a sequence, there exists $h_{i} \in L^{2}\left(0, T ; H^{2}(\Omega)\right),(i=1,2)$ such that $\nabla h_{i}$ and $\nabla \sqrt{h_{i}}$ belong to $L^{\infty}\left(0, T ; L^{2}(\Omega)^{2}\right)$ and $h_{i_{n}} \longrightarrow h_{i} \quad$ in $L^{p}\left(0, T ; H^{1+1 / p}(\Omega)\right) \cap \mathcal{C}\left([0, T] ; H^{1 / p}(\Omega)\right), \quad \forall p \in(2,+\infty)$. Next, since $\sqrt{h_{i_{n}}} v_{i_{n}}$ is bounded in $L^{\infty}\left(0, T ;\left(L^{2}(\Omega)\right)^{2}\right)$, we deduce that it converges weakly in $L^{2}\left(0, T ;\left(L^{2}(\Omega)\right)^{2}\right)$ up to a subsequence to some limit $z_{i} \in L^{2}\left(0, T ;\left(L^{2}(\Omega)\right)^{2}\right)$.

Let us define $v_{i}$ to be:

$$
v_{i}= \begin{cases}\frac{z_{i}}{\sqrt{h_{i}}} & \text { if } h_{i}>0 \\ 0 & \text { if } h_{i}=0 .\end{cases}
$$

To prove the convergence of $\left(h_{i_{n}} v_{i_{n}}\right)$, we write it as $h_{i_{n}} v_{i_{n}}=\sqrt{h_{i_{n}}} v_{i_{n}} \sqrt{h_{i_{n}}}$. Notice that $\sqrt{h_{i_{n}}} v_{i_{n}} \rightarrow$ $z_{i}$ in $L^{2}\left(0, T ;\left(L^{2}(\Omega)\right)^{2}\right)$, and so, we prove now the strong convergence for $\left(\sqrt{h_{i_{n}}}\right)$ in $L^{p}\left(0, T ; L^{p}(\Omega)\right)$. This proof is given as follows: we have $\left\|\nabla \sqrt{h_{i_{n}}}\right\|_{L^{2}\left(0, T ;\left(L^{2}(\Omega)\right)^{2}\right)} \leq C$ and $\left\|\sqrt{h_{i_{n}}}\right\|_{L^{\infty}\left(0, T ;\left(L^{4}(\Omega)\right)^{2}\right)} \leq C$, so, we can write that $\left(\sqrt{h_{i_{n}}}\right)$ is bounded in $L^{\infty}\left(0, T ; H^{1}(\Omega)\right)$ and $\partial_{t} \sqrt{h_{i_{n}}}=\frac{1}{2} \sqrt{h_{i_{n}}} \operatorname{div} v_{i_{n}}-\frac{1}{2} \operatorname{div}\left(\sqrt{h_{i_{n}}} v_{i_{n}}\right)$ is bounded in $L^{2}\left(0, T ; H^{-1}(\Omega)\right)$. Consequently, the subsequence, $\left(\sqrt{h_{i_{n}}}\right)$ converges strongly to some $\sqrt{h_{i}}$ in $L^{p}\left(0, T ; L^{p}(\Omega)\right)$. We then deduce that

$$
h_{i_{n}} v_{i_{n}} \rightarrow \sqrt{h_{i}} z_{i}=h_{i} v_{i} \quad \text { in } L^{2}\left(0, T ; L^{2}(\Omega)^{2}\right) .
$$

So we can pass to the limit in the mass equations. We give now some strong convergences which are necessary to pass to the limit in the momentum equations, more precisely in the frictions terms. For the others terms, see [7].

Additional convergence. Note that $\left|\delta_{n}^{-1}-\delta^{-1}\right| \leq \mu_{2}\left(\left|\gamma_{n}\right|\left|h_{1_{n}}-h_{1}\right|+\left|h_{1}\right|\left|\gamma_{n}-\gamma\right|\right)+\mu_{1}\left(\left|\gamma_{n}\right|\left|h_{2_{n}}-h_{2}\right|+\left|h_{2}\right|\left|\gamma_{n}-\gamma\right|\right)$. These terms have all them the same convergence behavior. So, by Holder inequality, we have 


$$
\int_{\Omega}\left|\gamma_{n}\right|^{p / 2}\left|h_{i_{n}}-h_{i}\right|^{p / 2} \leq \int_{\Omega}\left|\gamma_{n}\right|^{p} \int_{\Omega}\left|h_{i_{n}}-h_{i}\right|^{p} \text { and } \int_{\Omega}\left|h_{i}\right|^{p / 2}\left|\gamma_{n}-\gamma\right|^{p / 2} \leq \int_{\Omega}\left|h_{i}\right|^{p} \int_{\Omega}\left|\gamma_{n}-\gamma\right|^{p} \text {. Since }\left(h_{i_{n}}\right) \text { and }
$$

$\left(\gamma_{n}\right)$ are bounded in $L^{p}\left(0, T ; L^{p}(\Omega)\right)$, we find the strong convergence of $\left(\delta_{n}^{-1}\right)$ to $\delta^{-1}$ in $L^{p / 2}\left(0, T ; L^{p / 2}(\Omega)\right)$.

Next we have $\left\|\frac{\gamma_{n}}{\sqrt{h_{2_{n}}}}-\frac{\gamma}{\sqrt{h_{2}}}\right\|_{L^{3}\left(0, T ; L^{3}(\Omega)\right)}^{3} \leq \int_{0}^{T} \int_{\Omega}\left(a_{1}+a_{2}+a_{3}+a_{4}\right)^{3} d x$, being $a_{1}=\frac{\rho_{2}}{\rho_{1}}\left|\sqrt{h_{2_{n}}}-\sqrt{h_{2}}\right|$, $a_{2}=\frac{\rho_{2}}{\rho_{1}} h_{1_{n}} h_{2}\left|\sqrt{h_{2_{n}}}-\sqrt{h_{2}}\right|, a_{3}=\frac{\rho_{2}}{\rho_{1}} h_{2}^{3 / 2}\left|h_{1_{n}}-h_{1}\right|, a_{4}=\frac{\rho_{2}}{\rho_{1}} h_{1} \sqrt{h_{2}}\left|h_{2}-h_{2_{n}}\right|$.

There exist some constant $M_{q} \geq 0(1 \leq q \leq 8)$ such that $\left|\sum_{i=1}^{4} a_{i}\right|^{3} \leq \sum_{i=1}^{4} \sum_{q=1}^{8} M_{q} a_{i}{ }^{q}$. It's enough to prove the strong convergence of $\int_{0}^{T} \int_{\Omega}\left|a_{i}\right|^{q}$ for all $1 \leq i \leq 4$ and $1 \leq q \leq 8$. More precisely, the difficulties appear only for $\mathrm{i}=2,4$. We give the proof of the convergence of $a_{2}$. The rest follows the same way. To do this, we write:

$$
\int_{0}^{T} \int_{\Omega}\left|h_{1_{n}} h_{2}\right|^{q}\left|\sqrt{h_{2_{n}}}-\sqrt{h_{2}}\right|^{q} \leq \int_{0}^{T}\left(\int_{\Omega}\left|h_{1_{n}} h_{2}\right|^{2 q}\right)^{1 / 2} \int_{0}^{T}\left(\int_{\Omega}\left|\sqrt{h_{2_{n}}}-\sqrt{h_{2}}\right|^{2 q}\right)^{1 / 2}
$$

for all $q>1$. Hence, $\int_{0}^{T} \int_{\Omega}\left|h_{1_{n}} h_{2}\right|^{q}\left|\sqrt{h_{2_{n}}}-\sqrt{h_{2}}\right|^{q} \leq\left\|h_{1_{n}} h_{2}\right\|_{L^{2 q}\left(0, T ; L^{2 q}(\Omega)\right)}\left\|\sqrt{h_{2_{n}}}-\sqrt{h_{2}}\right\|_{L^{2 q}\left(0, T ; L^{2 q}(\Omega)\right)}$. But $\left\|h_{1_{n}} h_{2}\right\|_{L^{2 q}\left(0, T ; L^{2 q}(\Omega)\right)}$ is bounded and $\left(\sqrt{h_{2_{n}}}\right)$ converges strongly to $\left(\sqrt{h_{2}}\right)$ in $L^{2 q}\left(0, T ; L^{2 q}(\Omega)\right)$. Finally, we get the strong convergence of $\left(\frac{\gamma_{n}}{\sqrt{h_{n_{n}}}}\right)$ to $\frac{\gamma}{\sqrt{h_{2}}}$ in $L^{3}\left(0, T ; L^{3}(\Omega)\right)^{3}$.

These convergences allow us to pass to the limit in the friction terms.

\section{Acknowledgements}

The research of G. Narbona-Reina to carry on this work was partially supported by the Spanish Government Research project MTM2009-07719 and the research of J. D. D. Zabsonré by the Seville University OTRI grant 2010-00387.

\section{References}

[1] Bresch D., Desjardins B.: Existence of weak solutions for 2d viscous shallow water equations and convergence to the quasi-geostrophic model. Comm. Math. Phys., 238(1,2), 211-223 (2003).

[2] Bresch D., Desjardins B.: On the construction of approximate solutions for the 2d viscous shallow water model and for compressible Navier-Stokes models. J. Math. Pure Appl., 86, 362-368 (2006).

[3] F. Flori, P. Orenga, M. Peybernes. Sur un problème de shallow water bicouche avec conditions aux limites de Dirichlet. C.R. Acad. Sci. Paris. Ser. I 340 (2005) 719-724.

[4] P. Fabrie, F. Marche. Another proof of stability for global weak solutions of $2 D$ degenerated shallow water models. J. Math. Fluid Mech. 11 (2009), no. 4, 536-551

[5] M. L. Munoz-Ruiz, F. J. Chatelon, P. Orenga, On a bi-layer shallow water problem. Nonlinear Anal. 4 (2003) $139-171$.

[6] Narbona-Reina G., Zabsonré J. D. D., Fernández-Nieto E., D. Bresch.: Derivation of a bi-layer Shallow-Water model with viscosity. Numerical validation. CMES, 43(1), 27-71 (2009).

[7] Zabsonré J. D. D., Narbona-Reina G.: Existence of a global weak solution for a $2 D$ viscous bi-layer Shallow Water model. Nonlinear Analysis: Real World Applications,10, 2971-2984 (2009). 